\title{
Eine Suche nach Ordnung in der Geschichte der Menschheit Über Eric Voegelins Schriften in deutscher Sprache
}

Inhalt

1. Voegelins Werk in Deutschland

2. Politische Religionen und moderne Gnosis 259

3. Die deutsche Ausgabe von Order and History 261

4. Vom kosmologischen Mythos zu Geschichte und Philosophie 264

5. Menschsein in der Geschichte, Meditation und Mystik 267

6. Ordnung, Politik und Politikwissenschaft 270

7. Hitler und Deutschlands politische Kultur $\quad 272$

8. Die Krise der Moderne $\quad 274$

$\begin{array}{ll}\text { Literatur } & 278\end{array}$

\section{Voegelins Werk in Deutschland}

Als Eric Voegelin vor fünfzig Jahren aus den Vereinigten Staaten nach München kam, um an der Ludwig-Maximilians-Universität als Gründungsdirektor das Institut für Politische Wissenschaft aufzubauen und die Politikwissenschaft in München zu etablieren, waren seit seiner Emigration 1938 lediglich fünf kleine Texte von ihm auf Deutsch erschienen. Die vor 1938 publizierten Schriften - teilweise sehr schwer zugänglich und thematisch an Diskussionen der Zwischenkriegszeit orientiert - waren weithin vergessen; und Voegelin selbst unternahm nach seiner Ankunft in München nichts, um sie der Vergessenheit zu entreißen. So lag bei der Übernahme seiner Professur 1958 keine deutschsprachige Schrift Voegelins vor, die seinen eigenen Ansatz in der Form repräsentierte, in der er ihn zu jener Zeit ausgearbeitet hatte. Immerhin erschienen schon bald nach seiner Ankunft in München die deutsche Übersetzung der 1952 publizierten New Science of Politics sowie ein kleines Büchlein mit dem Text seiner Münchener Antrittsvorlesung und einer Studie über den Gottesmord (Voegelin 1959a, 1959b).

Die beiden Bücher von 1959 prägten für lange Zeit und bis in unsere Tage hinein das Bild, das man sich in Deutschland von Voegelin machte, wobei die Rezeption von Anfang an selektiv war: Voegelin galt hierzulande in erster Linie als radikaler Modernitätskritiker, der die Neuzeit als gnostisches Zeitalter des Verfalls betrachtete, dessen intellektuelle Repräsentanten wie Comte, Hegel oder Marx den Weg in die Totalitarismen des 20. Jahrhunderts gebahnt hätten. Auch wenn die Gnosisthese ein keineswegs beiläufiger Topos des Voegelin'schen 
Denkens der fünfziger Jahre war und die Modernitätskritik ein bestimmender Zug seines Werkes darstellt, ließ sich Voegelins Ansatz doch unmöglich hierauf reduzieren. Das hätte nicht nur eine genauere Lektüre jener Bücher bereits offenbaren können, sondern nicht zuletzt ein Blick in die damals vorliegenden Bände der großen Studie, die Voegelin kurz vor seiner Rückkehr nach Europa in den USA veröffentlicht hatte, nämlich die ersten drei seines auf sechs Bände angelegten Hauptwerkes Order and History. Diese englischsprachigen Arbeiten wurden aber in Deutschland, zumal in der deutschen Politikwissenschaft, so gut wie überhaupt nicht zur Kenntnis genommen (Opitz 1989; Weiss 2003: 29-38).

Die mangelnde Rezeption des Voegelin'schen Werkes selbst während der Zeit seines Wirkens in Deutschland dürfte zumindest in der Politikwissenschaft nicht allein der Tatsache geschuldet gewesen sein, dass englischsprachige Literatur etwas außerhalb des Fokus deutscher Wissenschaftler lag. Jedenfalls wurde auch Voegelins 1966 in deutscher Sprache veröffentlichtes und erst später ins Englische übersetzte Buch Anamnesis, in dem er seinen - inzwischen weiterentwickelten - Ansatz ausführlich und programmatisch entfaltet, weithin ignoriert. Man wird daher nach inhaltlichen Gründen für die nur ,,marginal verbliebene Rezeption“" (siehe Lübbe 2003) Voegelins in Deutschland zu fragen haben. Will man entsprechenden Fragen nachgehen, wird man nicht umhin kommen, sich mit Voegelins Werk im Ganzen auseinanderzusetzen. Das aber ist in Deutschland heute sehr viel leichter als zu Lebzeiten Voegelins, der nach seinem erneuten Weggang in die USA (1969) wieder vorwiegend englisch publizierte: Alle seine wichtigen Schriften, die zuerst auf Englisch erschienen, insbesondere Order and History, liegen jetzt in deutschen Übersetzungen vor. Daneben ist nun auch eine größere Zahl von Arbeiten sowohl aus seiner deutschen wie aus seinen amerikanischen Schaffensperioden zugänglich, die Voegelin selbst nicht publizierte; so namentlich einzelne Teile seiner monumentalen History of Political Ideas aus den vierziger Jahren und seine damals aufsehenerregende Vorlesung von 1964 über Hitler und die Deutschen. Ferner sind die wichtigsten seiner auf deutsch erschienen Schriften inklusive einer Reihe von Aufsätzen neu aufgelegt worden.

Dass Voegelins Werk dem deutschsprachigen Leser heute in dieser Weise zur Hand ist, ist zuallererst das Verdienst des Voegelin-Schülers Peter J. Opitz und des von diesem seit Jahren geleiteten Eric-Voegelin-Archivs an der Universität München. ${ }^{1}$ Schon wenige Jahre nach Voegelins Tod hatte Opitz beim Verlag Klett-Cotta eine Sammlung von späten Schriften besorgt (Voegelin 1988b), und 1991 gab er beim Alber-Verlag die vierte deutsche Auflage der New Science heraus (Voegelin 1991). Ebenfalls Anfang der neunziger Jahre konnte Opitz die Periagoge-Reihe beim Fink-Verlag initiieren, in der seither zum einen Schriften Voegelins (Periagoge - Texte) und zum anderen Forschungsarbeiten (vornehmlich) zu Voegelins Werk (Periagoge - Studien) publiziert werden. Deutschsprachige Schriften von und über Voegelin erschienen in jüngerer Zeit ferner im Wiener Passagen-Verlag, im Berliner Verlag Matthes und Seitz, erneut beim Alber-Verlag, und auch der österreichische Springer-Verlag hat ein

1 Zum Archiv siehe www.lrz-muenchen.de/ voegelin-archiv/. Opitz ist vermutlich der beste Kenner der Entwicklung des Voegelin'schen Denkens. Seine einschlägigen Studien, die er in Form von Vor- und Nachworten den Veröffentlichungen der Voegelin-Texte beigefügt hat, stellen einen inzwischen beachtlichen Korpus an Material für eine noch ungeschriebene bio-bibliografische Arbeit über Voegelins Leben und Werk dar, von der zu hoffen wäre, dass Opitz sie dereinst vorlegt. 
wichtiges frühes Werk Voegelins, den Autoritären Staat von 1936, neu herausgebracht (Voegelin 1997). Schließlich erscheinen seit 1996 Schriften von und über Voegelin in der Occasional-Papers-Reihe, die (nunmehr als „Voegeliniana“) vom Eric-Voegelin-Archiv herausgegeben wird. Im Folgenden werden einige der in neuerer Zeit auf deutsch publizierten Schriften Voegelins, insbesondere Ordnung und Geschichte, vorgestellt. Dabei wird auch der oben angesprochenen Frage nach den sachlichen Gründen der mangelnden Rezeption Voegelins nachgegangen - nicht zuletzt in der Absicht aufzuzeigen, dass eine Nichtbeachtung Voegelins in der Politikwissenschaft einerseits eine bedauerliche Selbstbeschränkung des Faches bedeutet, dass sie aber andererseits ein in gewisser Weise unvermeidliches Resultat von Mängeln des Voegelin'schen Ansatzes darstellt. ${ }^{2}$

\section{Politische Religionen und moderne Gnosis}

Den Auftakt der Periagoge-Reihe des Fink-Verlages bildete 1993 die Neuausgabe der Voegelin'schen Schrift über Die politischen Religionen (Voegelin 1993). Die Wiederveröffentlichung dieser zuerst 1938 erschienenen Schrift fiel in eine Zeit, in der nach dem Zusammenbruch des Ostblocks und dem Ende des Ost-West-Konfliktes die Totalitarismustheorie in der Politikwissenschaft erneut intensiv diskutiert und nach der Eigenart totalitärer Herrschaft gefragt wurde. ${ }^{3}$ Voegelins Konzept der politischen Religionen wurde dabei verschiedentlich als ein Angebot zum Verständnis totalitärer Bewegungen und Regime in konstruktiver Absicht aufgegriffen, namentlich um eine Erklärung für die Attraktivität zu finden, welche totalitäre Bewegungen für die ,politisierten“ Massen immer wieder gewinnen konnten. Dementsprechend gewann Voegelins Büchlein einige Aufmerksamkeit, weshalb 1996 eine zweite Auflage der Neuausgabe notwendig wurde, der nunmehr eine dritte Auflage folgte (Voegelin 2007a). Für diese hat Peter J. Opitz sein Nachwort überarbeitet, das inzwischen umfangreicher ist als Voegelins eigener Text (2007a). Opitz zeichnet darin die Genese der Voegelin'schen Studie minutiös nach und verortet sie im Gesamtwerk. Leider aber geht er hier nicht auf die Rezeption des Voegelin'schen Konzeptes innerhalb der jüngeren Totalitarismusdebatte ein. Damit wurde eine Gelegenheit vergeben, die Bedeutung, die Voegelins Konzept für eine wichtige zeitgenössische Diskussion hat, einmal zusammenfassend darzulegen - und zugleich auszuweisen, welches Anregungspotenzial Voegelins Werk für die zeitgenössische Politikwissenschaft haben kann.

Eine solche resümierende Darstellung wäre heute möglich, nachdem die Diskussion um das Konzept der politischen Religionen zu einem gewissen Abschluss gekommen zu sein scheint. Erinnert werden kann hier nur daran, dass im Rahmen eines großen, von Hans

2 Den Stand der internationalen Voegelin-Forschung vermitteln in repräsentativer Weise die Beiträge in Hughes/McNight/Price (2001) oder die Studien, die im Rahmen der Occasional-Papers-Reihe des EricVoegelin-Archivs veröffentlicht sind (Bergbauer 2002). Eine umfassende, bis zum Jahre 2000 reichende Bibliografie der Voegelin'schen Werke und der Sekundärliteratur ist Price 2000; diese Zusammenstellung wird ergänzt und fortgeführt in Opitz 2005. Zur Entwicklung des Voegelin'schen Euvres Henkel 1998; Opitz 2002.

3 Siehe etwa Backes/Jesse 1992; Söllner/Walkenhaus/Wieland 1997; Siegel 1998. 
Maier geleiteten interdisziplinären Forschungsprojektes die Tragfähigkeit des Konzeptes vor allem im Vergleich zur Totalitarismustheorie hinterfragt wurde. ${ }^{4}$

Voegelin selbst war mit dem Konzept der politischen Religion bald nicht mehr zufrieden und gab es als Kategorie geistesgeschichtlicher Forschung bald nach 1938 preis (Opitz 2007a: 135 f.). Eine differenziertere Weiterentwicklung des Konzeptes stellte der von Voegelin später für einige Jahre favorisierte Begriff der Gnosis dar, dessen Verständnis er 1951 in den Walgreen Lectures entwickelte, die als New Science of Politics bzw. als Die Neue Wissenschaft der Politik erschienen - Voegelins am weitesten verbreitetes Werk.

Die deutsche Erstausgabe von 1959 erfuhr 1965 eine unveränderte zweite Auflage. Eine dritte Auflage kam 1977 heraus. Sie enthielt die Textfassung der Vorauflagen, war aber neu gesetzt und hatte daher eine veränderte Paginierung. Die vierte Auflage von 1991 war im Text ein photomechanischer Nachdruck der dritten, während die Neuausgabe von 2007 abermals ein neues Druckbild und eine andere Seitenzählung aufweist. Somit existieren bei insgesamt fünf Auflagen drei Varianten desselben Textes mit unterschiedlicher Paginierung, was zu einer undurchsichtigen Situation für die Forschung führt. Die Ergänzung der jüngsten Fassung um eine Seitenkonkordanz wäre daher eine sinnvolle Hilfe gewesen, deren Fehlen um so mehr auffällt, als die Ausgabe ansonsten sehr großzügig ausgestattet ist - sie enthält ein Verzeichnis der Schriften Voegelins sowie ein umfangreiches und überaus hilfreiches Nachwort des Herausgebers, der dort auch das Gnosis-Konzept erläutert (Opitz 2007b: 249-258).

Das Konzept der Gnosis, das ursprünglich bestimmte (spät-)antike religiöse Strömungen bezeichnet, meint bei Voegelin die Auffassung, dass die Geschichte, ja die Realität als ganze in abschließender Weise erkannt werden können und dass aus solcher Erkenntnis ein Wissen um den Sinn der Geschichte erwachse. Mithilfe eines solchen Wissens würde die Geschichte oder die Realität durch Handeln in der Welt bewältigt werden können, und zwar namentlich durch ihre Überwindung. Gnosis ist in diesem Verständnis ein Erlösungswissen, näherhin ein - für Voegelin allerdings gar nicht erreichbares und in seinem Anspruch daher verfehltes - Wissen um die Selbsterlösungsmöglichkeit des Menschen. ${ }^{5}$ Im Unternehmen der Gnosis erkennt Voegelin die Re-Divinisierung, die Wiedervergöttlichung der Welt (Voegelin 2007b: 119, 135) - ein Prozess, der, gewachsen auf dem Boden des Christentums, un- und antichristlichen Charakters ist. Die Re-Divinisierung und nicht etwa die „Entzauberung“ (Max Weber) gilt Voegelin als das „Wesen der Modernität“ (119): Am Projekt der gnostischen Re-Divinisierung arbeiten Voegelin zufolge Bewegungen wie Aufklärung, Progressivismus, Liberalismus und Positivismus nicht weniger als Marxismus und Nationalsozialismus, die nur totalitäre Endstufen des gnostischen Selbsterlösungsprojektes darstellen (142).

Auch wenn Voegelin die Ambivalenz geschichtlicher Entwicklungen anerkennt und die Tatsache fortschrittlicher, gewissermaßen aufsteigender historischer Linien keineswegs leugnet (138-142), gilt ihm die Geschichte der westlichen Moderne im Ganzen als geistige

Maier 1995, 1996, 2003; Maier/Schäfer 1997; Henkel 1998: 86-91.

5 „Das Entscheidende ist, daß nach Voegelin gnostische Bewegungen eines gemein haben, nämlich die Vergegenständlichung der Realität als ganzer, die dadurch zum Gegenstand eines Wissens wird, das, je nach der Grundhaltung des Gnostikers, zur Befreiung von der Welt oder zu ihrer Veränderung führen muß“ (Hollweck 1999: 145). Siehe zur Gnosisthese Voegelins ferner Henkel 1998: 92-117; Opitz 1999; Seitschek 2003. 
Verfallsgeschichte. Ihr voraus geht eine Geschichte des zivilisatorischen Aufstiegs von den mythischen Weltbildern zur Entdeckung der Transzendenz in der griechischen Philosophie und der jüdisch-christlichen Offenbarung. Letztere stellt den Höhepunkt der geistigen Entwicklung, nämlich die höchste Differenzierungsform des menschlichen Geistes, des humanen Selbstverständnisses und zugleich der adäquaten Seinserkenntnis dar. Die jüdisch-christliche Offenbarung bildet in Voegelins Konzeption der Neuen Wissenschaft gewissermaßen den Scheitelpunkt der Geschichte (siehe bes. $2007 \mathrm{~b}$ : 172 f.).

\section{Die deutsche Ausgabe von Order and History}

Die Neue Wissenschaft der Politik erweist sich primär als eine Geschichtsphilosophie. Deren theoretischer Kern bildet eine Theorie der Repräsentation, mittels derer Voegelin historische Gesellschaften in ihrem Verhältnis zur Entfaltung des Geistes und zum entsprechenden Selbstverständnis der Menschen beurteilen kann. ${ }^{6}$ Es ist diese Geschichtsphilosophie, die die programmatische Grundlage von Order and History in der Konzeption darstellte, der die drei ersten, 1956 und 1957 erschienenen Bände verpflichtet waren. Zu Recht bezeichnete daher Helmut Kuhn Order and History als ,im Hinblick auf die christliche Heilsgeschichte entworfene Geschichtsphilosophie“ (Kuhn 1960: 125; Voegelin 2005a: 50). Indes wurde Voegelin im weiteren Verlaufe seiner Studien in den sechziger Jahren zu einer sehr grundlegenden Revision seines Ansatzes geführt, sodass der vierte Band von Order and History, der erst 1974 erschien, dem Geschichtsschema des ursprünglichen Konzeptes nicht mehr folgte und es in erheblichem Maße relativierte. Der 1987 postum veröffentlichte fünfte und nunmehr letzte Band des Unternehmens, In Search of Order, stellte abermals einen Neuanlauf dar, die Philosophie von Ordnung und Geschichte zu entfalten, ein Anlauf, der in Kontinuität vor allem zum vierten Band stand, sich in Anlage und Form aber gleichwohl von den anderen Bänden erheblich unterschied, indem er sich im Wesentlichen als eine mystische Meditation präsentierte.

Die Herausgeber des Gesamtwerkes, Peter J. Opitz und Dietmar Herz, haben sich entschieden, Voegelins Studie in zehn Teilbänden zu publizieren. Dies war zweifellos sinnvoll, nicht allein, weil eine der englischen Ausgabe entsprechende fünfbändige Edition Bücher jenseits eines vertretbaren Volumens hervorgebracht hätte ${ }^{7}$ sondern weil so mehr Raum dafür blieb, den Voegelin'schen Text um textkritische Bemerkungen zu den Übersetzungen sowie um erläuternde Nachworte zu ergänzen. Die Bände 1 bis 9 enthalten Personen- und Sachregister, Band 10 nur ein Personenverzeichnis. Die Bände der englischen und der deutschen Fassung sind folgendermaßen aufgeteilt und betitelt:

6 Zu Voegelins Repräsentationstheorie siehe Pitkin 1972: 44-47; Henkel 1998: 117-126; Opitz 2007b: 239242; Duso 2006: 200-226.

7 Dabei haben die Bände der deutschen Ausgabe teilweise noch immer einen stattlichen Umfang. Der (innerhalb des Gesamtwerkes umfangreichste) Platon-Band (2002c) etwa hat 389 Seiten, wovon 299 Seiten auf Voegelins Text kommen, der im dritten Band des Platon und Aristoteles behandelnden Originals 265 von 372 Seiten umfasst. 


\begin{tabular}{|c|c|}
\hline Order and History (1956-1987) & $\begin{array}{l}\text { Ordnung und Geschichte (2001-2005), hrsg. von } \\
\text { Peter J. Opitz und Dietmar Herz in Verbindung } \\
\text { mit dem Eric-Voegelin-Archiv an der Ludwig- } \\
\text { Maximilians-Universität München }\end{array}$ \\
\hline \multirow[t]{3}{*}{ Vol. I: Israel and Revelation (1956) } & $\begin{array}{l}\text { Bd. 1: Die kosmologischen Reiche des Alten } \\
\text { Orients: Mesopotamien und Ägypten, hrsg. von } \\
\text { Jan Assmann (2002) }\end{array}$ \\
\hline & $\begin{array}{l}\text { Bd. 2: Israel und die Offenbarung: Die Geburt } \\
\text { der Geschichte, hrsg. von Jörg Jeremias und } \\
\text { Friedhelm Hartenstein (2005) }\end{array}$ \\
\hline & $\begin{array}{l}\text { Bd. 3: Israel und die Offenbarung: Moses und } \\
\text { die Propheten, hrsg. von Jörg Jeremias und } \\
\text { Friedhelm Hartenstein (2005) }\end{array}$ \\
\hline \multirow[t]{2}{*}{ Vol. II: The World of the Polis (1957) } & $\begin{array}{l}\text { Bd. 4: Die Welt der Polis: Gesellschaft, Mythos } \\
\text { und Geschichte, hrsg. von Jürgen Gebhardt } \\
\text { (2002) }\end{array}$ \\
\hline & $\begin{array}{l}\text { Bd. 5: Die Welt der Polis: Vom Mythos zur Phi- } \\
\text { losophie, hrsg. von Jürgen Gebhardt (2003) }\end{array}$ \\
\hline \multirow[t]{2}{*}{ Vol. III: Plato and Aristotle (1957) } & Bd. 6: Platon, hrsg. von Dietmar Herz (2002) \\
\hline & $\begin{array}{l}\text { Bd. 7: Aristoteles, hrsg. von Peter J. Opitz } \\
\text { (2001) }\end{array}$ \\
\hline \multirow[t]{2}{*}{ Vol. IV: The Ecumenic Age (1974) } & $\begin{array}{l}\text { Bd. 8: Das Ökumenische Zeitalter: Die Legitimi- } \\
\text { tät der Antike, hrsg. von Thomas Hollweck } \\
(2004)\end{array}$ \\
\hline & $\begin{array}{l}\text { Bd. 9: Das Ökumenische Zeitalter: Weltherr- } \\
\text { schaft und Philosophie, hrsg. von Manfred } \\
\text { Henningsen (2004) }\end{array}$ \\
\hline Vol. V: In Search of Order (1987) & $\begin{array}{l}\text { Bd. 10: Auf der Suche nach Ordnung, hrsg. von } \\
\text { Paul Caringella und Gilbert Weiss (2004) }\end{array}$ \\
\hline
\end{tabular}

Die deutsche Übersetzung darf im Großen und Ganzen als geglückt betrachtet werden. Den insgesamt zwölf Übersetzerinnen und Übersetzern des Gesamtwerkes ist es weithin gelungen, Voegelins bisweilen eigenwillige Sprache und Terminologie angemessen wiederzugeben, wobei für die Terminologie auf die unverzichtbare Einheitlichkeit der Übersetzung in allen Teilbänden geachtet wurde. Ein jedem Band beigegebener textkritischer Apparat erläutert Details der Übersetzungen und gibt verschiedene Auskünfte über Voegelins 
Wortgebrauch, Schwierigkeiten der Übersetzung etc. Auch die erkennbar große Sorgfalt konnte indes nicht verhindern, dass bisweilen Übersetzungs- und Formulierungsfehler unterliefen, die gelegentlich zu sogar erheblichen Entstellungen oder Verzerrungen des von Voegelin gemeinten Sinns führten.

Dass Voegelins Werk nicht ohne erläuternde Aufsätze herausgegeben werden konnte, versteht sich von selbst. Dementsprechend finden sich in allen Bänden kommentierende Nach- und teilweise auch Vorworte der jeweiligen Bandherausgeber. Eine Ausnahme macht hierbei der in dieser Hinsicht besonders gelungene und vorbildliche erste Band über die altorientalischen Reiche Mesopotamiens und Ägyptens. Er enthält zunächst neben dem Voegelin'schen Text ein Vor- sowie ein Nachwort des überaus glücklich gewählten Bandherausgebers, des Ägyptologen und Kulturwissenschaftlers Jan Assmann. Assmann - ein renommierter und über die Grenzen der Fachwissenschaft hinaus bekannter Vertreter seiner Forschungsgebiete - kommt akademisch nicht von Voegelin, hat sich aber in seinen Arbeiten verschiedentlich mit Voegelin auseinandergesetzt und sich von diesem anregen lassen. Aus der Perspektive des Fachgelehrten beurteilt er die Voegelin'sche Darstellung des antiken Ägyptens, wobei er eine nüchterne, wohlwollend-kritische Distanz wahrt. So erhalten die Leser hilfreiche Hinweise darauf, wie sie in der Auseinandersetzung mit Voegelin ihrerseits eine differenzierte inhaltliche Position gewinnen können. Neben den Texten Assmanns findet sich zudem ein Text des Harvard-Gelehrten Peter Machinist, Professor für altorientalische Sprachen und Kenner der Geschichte und Literatur der antiken Reiche des vorderen Orients, der Voegelins Darlegungen über Mesopotamien diskutiert (Assmann 2002; Machinist 2002). Auch Machinists Text vermittelt dem Leser eine ausgezeichnete Grundlage zur Beurteilung der Voegelin'schen Studie. Schließlich enthält der Band einen Beitrag von Opitz, der die Genese von Ordnung und Geschichte im Einzelnen darlegt, und so dem Eröffnungsband der deutschen Ausgabe einen gründlichen Überblick über Voegelins intellektuelle Entwicklung beigibt (Opitz 2002).

Ähnlich wie mit dem ersten verhält es sich auch mit den Bänden zwei und drei: Diese wurden von den Alttestamentlern Jörg Jeremias und Friedhelm Hartenstein herausgegeben und kommentiert, also von Gelehrten, die die entsprechenden Gegenstände der Voegelin'schen Studien in ihrer Forschung bearbeiten und aus der Perspektive ihres Fachgebietes die Darlegungen des alttestamentlichen Autodidakten Voegelins in den Blick nehmen.

Sowohl Assmann als auch Machinist, Jeremias und Hartenstein bescheinigen Voegelin gründliche Kenntnisse und einen bemerkenswerten Sensus für die behandelten Themen - ungeachtet der Kritik im Einzelnen und ungeachtet der Tatsache, dass Voegelins Studien vielfach nicht dem heutigen Stand der Fachwissenschaften entsprechen können. So bemerken etwa Hartenstein und Jeremias, dass Voegelins Israelstudie ,auch für heutige Leser eine beispielhafte kulturwissenschaftliche Untersuchung“ darstelle. „,Trotz ihrer Korrekturbedürftigkeit in manchen Einzelheiten“, heißt es weiter, ,entwirft sie ein ebenso eigenständiges wie treffendes Gemälde der Selbstinterpretation des alten Israel“ (Hartenstein/Jeremias 2005: 11).

Gemessen an der Gestaltung der ersten Bände fallen mit Blick auf die kommentierenden Vor- und Nachworte die Bände vier bis zehn leider zurück. Es wäre hilfreich gewesen, auch diesen Bänden entsprechende Nachworte von Fachwissenschaftlern beizugeben. So wäre eine Beurteilung der Bände über Das Ökumenische Zeitalter durch einen umfassend gebildeten 
Michael Henkel

Historiker wie etwa Alexander Demandt oder einen historisch arbeitenden Soziologen wie den Achsenzeit-Forscher Shmuel N. Eisenstadt sicher nicht weniger aufschlussreich gewesen als eine Kommentierung der Voegelin'schen Interpretation chinesischer Ordnungsvorstellungen (Voegelin 2004b: 139-172) durch einen Kenner der älteren chinesischen Geschichte und der chinesischen Philosophie. ${ }^{8}$ Namentlich für die Bände über Platon und Aristoteles hätten sich die Philologen bzw. Philosophen Norbert Blößner, Hellmut Flashar, Christoph Horn und Eckart Schütrumpf als Kommentatoren schon deshalb angeboten, weil diese sich als renommierte Aristoteles- und Platonforscher im Rahmen der erwähnten Occasional-Papers-Reihe (Flashar auch andernorts) mit Voegelins Interpretationen grundlegend auseinandergesetzt haben ${ }^{9}$ - immer vorausgesetzt, die Genannten hätten eine Herausgebertätigkeit oder eine Kommentierung übernommen. Sicher orientieren die tatsächlich beigegebenen Nachworte der jeweiligen Bandherausgeber mehr oder weniger gründlich über Voegelins Intention und Argumentation. Die weitgehende Beschränkung der entsprechenden Ausführungen auf die - in zwei Fällen mit hagiographischen Untertönen, in einem Fall als langatmige und etwas ermüdende Nacherzählung präsentierte - Darstellung der Voegelin'schen Sicht lässt aber das vergleichende Urteil aus der Perspektive der jeweiligen Fachforschung vermissen.

\section{Vom kosmologischen Mythos zu Geschichte und Philosophie}

Die ersten drei Bände von Order and History folgen in ihrer Darstellung dem geschichtsphilosophischen Schema, das Voegelin in der Neuen Wissenschaft dargestellt hatte und das den Plan des Gesamtprojektes in seiner ursprünglich beabsichtigten Form prägte. Demnach sollte das Werk sechs Bände umfassen, nämlich neben den drei tatsächlich vorgelegten die Bände (IV.) Empire and Christianity, (V.) The Protestant Centuries und (VI.) The Crisis of Western Civilization (Voegelin 2002a: 28). Die ersten vier Bände hätten demnach den geistigen Aufstiegsprozess hin zur maximal differenzierten Seinserfahrung und ihrer Symbolisierung zum Thema gehabt, während die übrigen Bände den geistigen Abstiegsprozess behandeln sollten, der für Voegelin durch die Herausbildung der westlichen Nationalstaaten ,und die Entwicklung der Gnosis als der symbolischen Ordnungsform“ (2002a: 28) charakterisiert war. In den sechziger Jahren allerdings sah sich Voegelin zunehmend genötigt, seine Konzeption erheblich zu modifizieren, sodass er den ursprünglichen Plan des Werkes aufgab und mit dem tatsächlich erschienen vierten Band einen Neuanlauf nahm.

Mithin liegen von dem ursprünglichen Projekt nur drei von den Bänden vor, die den - im Wesentlichen linear gedachten - geistig-historischen Differenzierungsprozess behandeln. Dieser wird nachgezeichnet im Ausgang von den Reichen des alten Orients (Voegelin 2002a), die die ihnen entsprechende kompakte Seinserfahrung im kosmologischen Mythos symbolisieren, hin zu der in Israel entfalteten Symbolform der durch Offenbarung konstituierten Geschichte (Voegelin 2005a, 2005b) und jener der in Griechenland geborenen, den Mythos überwindenden Philosophie (Voegelin 2001, 2002b, 2002c, 2003a). Den Symbolformen

8 Als Sinologe und ausgewiesener Kenner der klassischen chinesischen Philosophie (Opitz 2000) hätte gewiss auch der Gesamtherausgeber Opitz einen entsprechenden Beitrag leisten können.

9 Blößner 2001; Flashar 2001, 2003, 2005; Horn 2001; Schütrumpf 2001. 
der Geschichte und der Philosophie liegt die Entdeckung der Transzendenz zugrunde, die in der Geschichte der Ordnung einen „Seinssprung“ (,leap in being“) bedeutet, welcher für die Ordnung der (nachfolgenden) Geschichte konstitutiv ist. ${ }^{10}$

Voegelin zeichnet die entsprechenden Prozesse anhand einer Vielzahl ausführlicher Interpretationen nach, wobei er die diskutierten Autoren, Werke und literarischen Zeugnisse stets in ihren historischen und politischen Kontext einordnet und aus diesem heraus in den Blick zu nehmen sucht, um die den Symbolformen zugrunde liegenden Ordnungserfahrungen greifen zu können und nicht in eine abstrakte Ideengeschichte zurückzufallen, die zu überwinden eine der wesentlichen Intentionen des Voegelin'schen Projektes war. In der Darstellung sucht er Nuancen und Differenzierungen in der Erfahrungswelt der Antike offenzulegen, um sichtbar werden zu lassen, wie sich der Durchbruch zur Transzendenz vorbereitete und wie sich in diesem Durchbruch der Mensch in seinem Menschsein und dieses Menschsein in seiner Universalität entdeckt. Es ist der tiefere Sinn des Voegelin'schen Werkes, das Menschsein des Menschen zu beleuchten, das Voegelin zwar als in der Natur des Menschen sich gleichbleibend gegeben betrachtet, das ihm aber zugleich als dem einzelnen Menschen und den historischen Gesellschaften immer wieder aufgegeben, als niemals eindeutig, ein für allemal fixierbar gilt. Die menschliche Existenz bleibt ein Mysterium, im Letzten unergründlich, und doch muss sich der Mensch zu seiner Natur verhalten und sein Leben führen. Wesentlich hierfür ist in Voegelins Augen, dass der Mensch erkennt, zwar in, aber keineswegs nur von dieser Welt zu sein (Voegelin 2002a: 39 ff.). Es ist die Teilhabe an der Transzendenz und die Einsicht in die für den Menschen existenzbegründende Spannung zwischen Transzendenz und Immanenz, durch die der Mensch zum Menschen wird; und in diesem Sinne heißt es etwa, dass „die Bestimmung des Menschen [...] in der Ewigkeit liege“ (Voegelin 2002b: 20).

Voegelins Suche nach Ordnung ist also - zumindest seit den fünfziger Jahren - in ihrem tiefsten Kern die Suche nach dem Menschsein des Menschen. Einen vergleichsweise unumwundenen Zugang zu diesem Kern bieten die von Voegelin 1967 gehaltenen Walter Turner Candler Lectures über Das Drama des Menschseins, die nunmehr vom Passagen-Verlag auf Deutsch publiziert wurden (Voegelin 2007c). Trotz einiger weniger Unklarheiten, die darauf zurückzuführen sind, dass es sich hier um eine von Voegelin allenfalls marginal überarbeitete Vorlesungsmitschrift handelt, kommt in diesem Buch sein Anliegen sehr deutlich zum Ausdruck. Wertvoll ist das Buch vor allem, weil es in einmalig konziser Form das Ordnungskonzept Voegelins in dessen eigenen Worten im Zusammenhang präsentiert.

Obgleich Voegelins geschichtsphilosophischer Konzeption der fünfziger Jahre zufolge das Menschsein des Menschen erst im Christentum seinen adäquaten symbolischen Ausdruck findet, ist es die Philosophie Platons, die das maßgebende Paradigma für die Symbole

10 „Der Seinssprung, das epochale Ereignis, das die Kompaktheit des frühen kosmologischen Mythos bricht und die Ordnung des Menschen unmittelbar unter Gott stellt, ereignet sich [...] zweimal in der Geschichte der Menschheit, ungefähr zur gleichen Zeit, im Nahen Osten und in den benachbarten Zivilisationen der Ägäis. Zwar laufen beide Ereignisse zeitlich parallel und haben die Opposition zum Mythos miteinander gemein, doch sind sie voneinander unabhängig; und die beiden Erfahrungen unterscheiden sich von ihrem Inhalt her so grundlegend voneinander, daß sie sich in den zwei verschiedenen Symboliken der Offenbarung und der Philosophie artikulieren“(Voegelin 2002b: 17; zum „Seinssprung“ ausführlich, 19-23). 
abgibt, welche die Erfahrung der existenziellen Spannung zum Ausdruck bringen. Dementsprechend ist Platon in allen zehn Bänden von Ordnung und Geschichte - in den ersten drei weithin nur implizit - präsent. Einer ausführlichen monografischen Darstellung der platonischen Philosophie ist dann der sechste Band gewidmet (Voegelin 2002c). Die Platonstudie offeriert eine ebenso tiefgehende wie im Ganzen verständlich geschriebene Darstellung der Philosophie Platons, die als eigenständige Studie für sich zu stehen vermag. Voegelin legt hier nach einer Diskussion des Verhältnisses von Platon zu Sokrates (17-40) akribische Interpretationen der Schriften Gorgias, Politeia, Phaidros, Politikos, Timaios, Kritias und Nomoi vor. Das Platon-Bild, das dabei gezeichnet wird, ist erkennbar dem Platonverständnis des Kreises um Stefan George verpflichtet, stimmt zudem aber in zentralen Punkten mit der jüngeren Tübinger Platonforschung überein, die Voegelin noch nicht kennen konnte (Flashar 2001: 7 ff.). ${ }^{11}$

Wie sehr Voegelin Platon verpflichtet ist, mag man schon dem äußerlichen Umstand ablesen, dass er der Interpretation Platons 299 Seiten widmet, während der Text des AristotelesBandes nur zwei Fünftel dieses Umfangs (120 Seiten) umfasst. Beide Teilbände lassen aber besonders schön hervortreten, dass Voegelin ein Meister begrifflicher Differenzierung und argumentativer Subtilität war. Diese Feststellung erhält ihr ganzes Gewicht erst durch den Vergleich der Voegelin'schen Interpretationen mit den allzu oft oberflächlichen, schematischen und ohne Problembewusstsein verfassten Darstellungen, die Platon und Aristoteles in einschlägigen politikwissenschaftlichen Lehrbüchern erfahren. Und dies gilt ungeachtet des Umstandes, dass man gegenüber Voegelins Platon- und Aristoteles-Interpretation z. T. gewichtige Einwände wird geltend machen müssen, wie sie etwa von Schütrumpf (2001: 24 f., 34 f., 57 f., 60, 62, 75, 80), Flashar (2001: 12-17, 21-23) oder Blößner (2001: 23-26) ausgewiesen wurden.

Als exemplarisch für Voegelins problemorientierte und differenzierte Darlegungen sei nur ein Aspekt seiner Aristoteleslektüre hervorgehoben: In seiner Exegese der Aristotelischen Verfassungstypologie stellt Voegelin (2001: 91-96) zunächst Aristoteles' Schema der sechs nach Herrscherzahl und Qualität unterschiedenen Verfassungsformen vor. Aristoteles folgend diskutiert er die entsprechende Typologie sodann aber im Zusammenhang der Gerechtigkeitsproblematik, durch deren praktische Ansprüche das abstrakte Schema unterlaufen und erweitert wird. Anders als manch gängige Lehrbuchdarstellung, die bei der Präsentation des Schemas endet, bekommt Voegelin die Pointe der Aristotelischen Ausführungen in den Blick, die darin besteht, dass erst die Empirie das abstrakte Schema politikwissenschaftlich zum Sprechen bringt.

11 Die „Hauptthese“ der Tübinger Platoniker ist, „,daß die Dialoge nur die Außenseite der platonischen Philosophie ausmachen, die ihren Kern in einer hinter den Dialogen stehenden, streng systematisch deduzierten Prinzipienlehre haben, welche Platon [...] von der schriftlichen Fixierung [...] ausgeschlossen hat. Es ist klar, daß der philosophische Status der Dialoge dadurch in einem neuen Licht erscheint. Darüber gibt es eine bis heute andauernde Kontroverse“ (Flashar 2001: 8). 


\section{Menschsein in der Geschichte, Meditation und Mystik}

Durch seine Studien der sechziger Jahre erkannte Voegelin, dass sich die Vorstellung eines im Wesentlichen linearen, durch eine intellektuelle Auf- und Abstiegslinie gekennzeichneten Geschichtsverlaufs jedenfalls nicht in der Weise halten ließ, wie es in den drei ersten Bänden von Order and History vorausgesetzt worden war. Die monografische Arbeit, in der sich die von Voegelin selbst als Bruch bezeichnete Neuausrichtung von Ordnung und Geschichte ankündigt und theoretisch vorbereitet wird, ist das Buch Anamnesis von 1966. Wie die Neue Wissenschaft gewissermaßen als Programmschrift der drei ersten, so kann Anamnesis als Programmschrift der beiden letzten Bände von Order and History angesehen werden. Auch diese Arbeit liegt nun in einer vom Alber-Verlag herausgebrachten Studienausgabe vor, die einen Nachdruck des Originals darstellt, ergänzt um ein Nachwort des Herausgebers Opitz (Voegelin 2005c).

Damit ist dasjenige Werk neu zugänglich, das nicht nur das wichtigste Bindeglied zwischen den ersten und den beiden letzten Bänden von Order and History darstellt, sondern das auch entscheidende Modifikationen im Stil des Voegelin'schen Denkens und dessen Präsentation gut erkennen lässt: Neben einer Reihe diskursiver Studien - beispielsweise über „das Rechte von Natur" (Voegelin 2005c: 117-133), über Bakunin (223-238) oder über John St. Mill (239-253) - stehen bewusstseinsphilosophische Meditationen (37-76, 283-354), die den Charakter des Bandes prägen und die für die Eigenart aller späteren Schriften Voegelins charakteristisch sind. Nicht zuletzt macht Anamnesis die Akzentverschiebungen in Voegelins Geschichtsphilosophie deutlich, die den Wandel in der Konzeption von Order and History nach sich ziehen, wie er im vierten Band zum Ausdruck kommt.

Zwar gab Voegelin die Vorstellung von Prozessen der Entwicklung vom kompakten zum differenzierten Bewusstsein und der entsprechenden Seins- und Ordnungserkenntnis keineswegs preis, aber er betonte nunmehr, dass diese Prozesse als Geschichte des Menschseins nicht mit einer linearen pragmatischen Universalgeschichte schlicht korreliert werden können, sondern sich chronologisch zu verschiedenen Zeiten in verschiedenem Ausmaß und geografisch an verschiedenen Orten der Welt ereigneten (dazu Henkel 1998: 153-168). Sicher hatte Voegelin in Anlehnung namentlich an Karl Jaspers' Konzept der Achsenzeit schon 1957 darauf verwiesen, dass der Bruch mit dem Mythos und der „Seinssprung“ sich etwa zur gleichen Zeit wie im Alten Orient und in Griechenland auch ,im Indien des Buddha und im China des Konfuzius und des Lao-tzu“ (Voegelin 2002b: 17) ereignet habe. Als Konsequenz gerade dieser frühen Einsicht ergab sich ihm aber in zunehmendem Maße, dass die Geschichte des Menschseins nicht länger „im Hinblick auf die christliche Heilsgeschichte“ (Kuhn 1960: 125) konzipiert werden konnte. Eine Analyse der Ordnungsgeschichte und der Geschichtsordnung, so schrieb Voegelin 1974 schließlich, habe sich „rückwärts, vorwärts und seitwärts“ zu bewegen, ,um empirisch den Bedeutungsmustern zu folgen, die sich in der eigenen Interpretation [eigentlich: Selbstinterpretation - im Original: self-interpretation; M. H.] von Personen und Gesellschaften in der Geschichte offenbarten“ (Voegelin 2004a: 83). Dieser Einsicht folgten die Darlegungen im vierten Band von Order and History, der nun eine Studie der spirituellen Ereignisse und Entwicklungen im antik-spätantiken Zeitalter der ökumenischen Reiche des Westens und des fernen Ostens präsentierte, in chronologischer 
Michael Henkel

Hinsicht also nicht über die Zeit der westlichen (Spät-)Antike hinausgriff, geografisch gesehen allerdings eine prinzipiell globale Perspektive einnahm.

Die Akzentverschiebung im Geschichtsbegriff bedeutet, dass Geschichte nunmehr radikal auf die Theophanie im individuellen Bewusstsein relativiert wird, genauer: auf die individuelle Erfahrung der existenziellen Spannung, eine Erfahrung, die in bestimmten historisch-gesellschaftlichen Umfeldern in für diese je paradigmatischer Weise auftreten kann: „Die theophanen Ereignisse geschehen nicht in der Geschichte; sie konstituieren die Geschichte mitsamt ihrem Sinn. Schließlich offenbart die noetische Theophanie, daß das Bewusstsein die Struktur der metaleptischen [teilhabenden; M.H.] Realität, des göttlichmenschlichen metaxy [i. e. des Zwischen; M.H.]“ - also der Existenzspannung zwischen Immanenz und Transzendenz - ,,besitzt“ (Voegelin 2004b: 114). ${ }^{12}$ Geschichte und ihre Sinnhaftigkeit werden also konstituiert in der Erfahrung der spannungshaften Teilhabe des Menschen an der Transzendenz. Hieraus zieht Voegelin weitreichende Folgerungen für den Geschichtsbegriff, so etwa, dass „das Geschichtsfeld immer universal-menschlich“ sei, weil „alle Menschen“ in der Suche nach dem transzendenten Grund „einander gleich sind“ (Voegelin 2005c: 299).

Die sinn- und geschichtskonstituierende Transzendenzerfahrung sieht Voegelin nun aber in den unterschiedlichsten kulturellen Symbolisierungen - wenngleich in verschiedenen Graden der Differenziertheit - zum Ausdruck gebracht, welche nicht in einen chronologischen Zusammenhang eingeordnet zu werden vermögen. Vor diesem Hintergrund sucht er nun ,rückwärts, vorwärts und seitwärts“ nach Äquivalenzen in den historischen Symbolformen, in denen jene Erfahrung zum Ausdruck kommt - und relativiert derart auch etwa die Grenze zwischen Mythos und Philosophie (Voegelin 1988a; 2004b: 36-41; 2005c: 299).

Obwohl Voegelin an der allgemeinen Relevanz der christlichen Offenbarung auch im vierten Band von Order and History bzw. den entsprechenden deutschen Teilbänden ausdrücklich festhält (Voegelin 2004b: 177 f.), wird der Geschichtsprozess der Menschheit von der welthistorischen Bedeutung Jesu Christi erkennbar abgelöst. Damit werden auch das Verständnis und die Stellung des Christentums im Werk Voegelins fragwürdiger, eine Problematik, die besonders in der US-amerikanischen Voegelin-Forschung kontrovers diskutiert wird. Die offenkundige Relativierung der Bedeutung des Christentums stellt im Grunde einen Aspekt des Voegelin'schen Bemühens dar, eine wirklich globale Perspektive einzunehmen und jegliche „kulturalistische“ Befangenheit zu überwinden.

Tatsächlich zeigt gerade der vierte Band Voegelin als Denker des globalen Zeitalters, der sich nicht dem Vorwurf aussetzen will, einem ,abendländischen Vorurteil“ (Voegelin 2002b: 23) oder einem sonstigen Provinzialismus verpflichtet zu sein. Dies wiederum ist letztlich eine Konsequenz des Unternehmens der Voegelin'schen Ordnungssuche: Das Menschsein des Menschen lässt sich nämlich nicht durch eine Verallgemeinerung partikularer Vorstellungen bestimmen. Vielmehr muss die Einsicht in das Eigentliche des Menschseins im unmittelbaren „Durchgriff“ zur Universalität des Menschseins, die dieses mitkonstituiert, erfolgen - also in

12 Gerade angesichts der Formulierung im vorstehenden Zitat ist es wichtig zu sehen, dass für Voegelin die Geschichte ausdrücklich keinen Sinn hat bzw. es nicht den einen Sinn der einen Geschichte gibt. Vielmehr gibt es Sinn bzw. Sinnlinien innerhalb der Geschichte bzw. Geschichten (Voegelin 2002b: 18; 2004b: 70, 80; 2005a: 43-51; 2005c: 321; 2007b: 131; Opitz 2007c: 152). 
dem als Spannung erfahrenen Bewusstsein des von Voegelin so bezeichneten (göttlichen) Grundes. Von dieser Spannung her erfährt der Mensch seine Ordnung und sein Maß, und diese sind es, von denen aus auch die gesellschaftliche Ordnung bestimmt werden muss.

Deshalb heißt es in Anamnesis programmatisch: „Die Probleme menschlicher Ordnung in Gesellschaft und Geschichte entspringen der Ordnung des Bewußtseins. Die Philosophie des Bewußtseins ist daher das Kernstück einer Philosophie der Politik. [...] Das Bewußtsein ist nicht ein Gegebenes, das von außen beschrieben werden könnte, sondern eine Erfahrung des Partizipierens am Seinsgrund, deren Logos nur durch meditative Exegese ihrer selbst zur Klarheit gebracht werden kann“(Voegelin 2005c: 7). Eine solche Exegese aber eröffnet eine Einsicht in das Menschsein des Menschen, in das Drama dieses Menschseins und in den Sinn der „Idee der Menschheit“ (99, 102 f.) bzw. des Symbols der einen Menschheit, das dem Verständnis des universalen Menschseins entwächst: „Ohne Universalität gäbe es Menschheit nur als die Gesamtmenge der Mitglieder einer biologischen Spezies“ (Voegelin 2004b: 179). ${ }^{13}$ Es ist damit die Erfahrung der Teilhabe an der universalen Transzendenz (dem universalen Gott), die den Einzelnen aus der Befangenheit in Stamm, Volk, Nation, Klasse oder Rasse etc. emanzipiert, die mithin den Menschen zu seinem Menschsein freisetzt und die universale Menschheit konstituiert. ${ }^{14}$

Voegelins Philosophie des Bewusstseins - das „Kernstück“ seiner Philosophie - lässt von Anfang an eine mystische Dimension erkennen und nimmt schließlich überhaupt die Form mystischer Meditation an. Deren reifster Ausdruck präsentiert der letzte Band des Hauptwerkes - Auf der Suche nach Ordnung. Darin legt Voegelin das - unabgeschlossen gebliebene - Zeugnis seiner späten meditativen Exegese ab, die ihm die Struktur des Bewusstseins und der Realität durchsichtig werden lässt. Was in diesem Buch vor sich geht, ist nicht leicht zu fassen (dazu Winterholler 2006); selbst die Herausgeber des Bandes bieten drei unterschiedliche und sich teilweise widersprechende Deutungen davon an, was das Thema der Studie sei: „die Möglichkeit der Sprache, der Sprache der Philosophen“ sei ,zentrales Thema“, heißt es zuerst; wenige Zeilen später wird „die paradoxe Struktur der Sprache“ (Caringella/Weiss 2004a: 15) zum Thema erklärt; schließlich aber wird dem Leser eröffnet, man könne als ,das Thema des Voegelin'schen Spätwerkes [...] das paradoxe Verhältnis von Erfahrung, Imagination und Symbolisierung“ (Caringella/Weiss 2004b: 156) nennen.

Keiner dieser Hinweise ist falsch, doch sie alle treffen vermutlich nicht den Kern der Sache. Voegelins Meditationen kreisen um die Erhellung des von ihm so genannten Komplexes „Bewußtsein-Realität-Sprache“ (Voegelin 2004c: 23 ff.) und der diesen Komplex repräsentierenden Symbole. Voegelin will damit den Logos der „Ordnung der Existenz des Menschen als eines Partners in der umfassenden Realität" (34) zur Sprache bringen, und dies dürfte er als einen „Akt der offenen Teilhabe am Prozeß der Geschichte und am Prozeß des Ganzen“ (Voegelin 2004b: 215) - welche den Prozess des Menschseins einschließen - begreifen. Es ist dies im Grunde eine auf das Ganze abhebende mystische Meditation über Sein und Zeit. ${ }^{15}$

13 Zur universalen Menschheit Voegelin 2004b: 173-215, 2007c: 52 ff.; Gebhardt 1982.

14 „Die Göttlichkeit des Geistes, die in allen anderen präsent ist, sie ist es, die den Menschen und die Würde des Menschen konstituiert und dazu verpflichtet, andere Menschen zu respektieren“ (Voegelin 2007c: 68).

15 Den Hinweis, dass „Ordnung und Geschichte“ auch für „Sein und Zeit“ stehen kann, gibt zu Recht Assmann 2002: 220. 
Michael Henkel

Wie alle Mystik kämpft auch diejenige Voegelins mit dem Problem, dass die (höchst individuelle) mystische „Erfahrung grundsätzlich jede menschliche Möglichkeit, davon zu reden [übersteigt]“ (Haas 2002: 77), und ebendeshalb widmet Voegelin der Sprache und ihren Paradoxien, die in gewisser Weise die Paradoxien der menschlichen Existenz widerspiegeln, so große Aufmerksamkeit. Aber letztlich geht es ihm um die Sprache nur, insofern sie die Erfahrung des Ganzen - deren Kern eine cognitio Dei experimentalis ist - nahebringen kann und soll. Das Mysterium des Ganzen - nichts Geringeres als dies ist es, worauf Voegelin dem Leser einen Ausblick gewähren will.

\section{Ordnung, Politik und Politikwissenschaft}

Wie immer man dieses meditative Projekt beurteilen mag - und niemand wird solcher Mystik ihr Recht bestreiten können -, erhebt sich die Frage, was die Herausgeber des abschließenden Bandes meinen, wenn sie versichern, Voegelin spreche hier ,als politischer Philosoph zu uns“" (Caringella/Weiss 2004b: 148). Was sie damit im Blick haben dürften, wird deutlich im letzten Satz ihres Nachwortes: Die politikwissenschaftliche „Relevanz“ der Voegelin'schen Meditationen erschließe sich, wenn die Politikwissenschaft „die Frage nach den philosophischen Ursprüngen von Ordnung und Herrschaft noch“ (174) zulasse. In dieser Perspektive wäre die marginal gebliebene Rezeption Voegelins von der Politikwissenschaft dadurch verschuldet, dass sie jene Frage nicht mehr zulasse.

Der Hinweis Caringellas und Weiss' knüpft in der Sache offenkundig daran an, dass Voegelin als das „Kernstück“ einer Theorie der Politik die Philosophie des Bewusstseins betrachtete, derzufolge sich das individuelle Bewusstsein als das „Zentrum“ der Ordnungserfahrung erweist (Voegelin 2005c: 8). Einsicht in die Eigenart, oder sogar in die Wahrheit bzw. Richtigkeit politischer Ordnung, also politikwissenschaftliche Einsicht, setzen demzufolge Ordnungserfahrungen - und das sind hier zu allererst Transzendenzerfahrungen - im individuellen Bewusstsein voraus. Mit dieser Auffassung macht sich Voegelin das anthropologische Prinzip Platons vollständig zu eigen, dass nämlich die Polis der großgeschriebene Mensch sei, mithin die Ordnung der Seele das Maß der Polis vorgebe (Voegelin 2002c: 112; 2001: 42; 2007b: 75-77).

Nicht zuletzt dieses Prinzip aber spricht der Sphäre der Politik ihre Eigenständigkeit ab: Politische Ordnung wird gewissermaßen zur unselbstständigen Funktion der seelischen Ordnung. Die wahre Ordnung der Seele wird zur Bedingung richtiger politischer Ordnung, seelische Unordnung zur Grundlage politischer Unordnung. Es ist vor diesem Hintergrund alles andere als ein Zufall, dass Voegelin durchgängig von individuell-menschlicher, geschichtlicher und sozialer Ordnung, kaum je von der unabhängigen politischen Ordnung spricht, ${ }^{16}$ die von der sozialen Ordnung spezifisch zu differenzieren ist. Tatsächlich erweist sich der Voegelin'sche Ordnungsbegriff als zu umfassend: Es mangelt ihm an einer Disziplinierung und Spezifizierung durch einen Begriff der Politik in dem Sinne, wie er im Zusammenhang

16 Besonders deutlich etwa die Rede von den „drei Dimensionen der Ordnung - persönlich, sozial, historisch“ (Voegelin 2004b: 177) - das Fehlen von ,politisch“ springt dem Leser hier geradezu in die Augen. Zum Politikverständnis Voegelins aufschlussreich Gebhardt 2003. 
der Entdeckung des Könnens-Bewusstseins im antiken Griechenland in die Welt gekommen ist (Meier 1995: 17, 45).

In der Sache aber bedeutet das nicht zuletzt eine Ausblendung der Eigenständigkeit der Politik, die als Konstitutivum anzuerkennen die Leistung nicht Platons sondern des Aristoteles ist: Für diesen ist die Polis ,ein der Art nach eigenes Gebilde [..., ] nicht die Seele in Großbuchstaben, wie Platon wollte. Damit gibt es bei Aristoteles auch zum ersten Mal politische Theorie im modernen Sinne, die diesen Namen verdient, und nicht etwa nur eine auf ein größeres Format projizierte Seelenkunde, wie bei Platon“ (Schütrumpf 2001: 86 f.). In diesem Sinne hat der „Vater der Politischen Wissenschaft in Deutschland“ (Mommsen 1962: 350), Hermann Heller, festgehalten, dass es ,einen eigentlichen Gegenstand der politischen Erkenntnis [erst dort] gibt [...], wo das Politische in seiner relativen Eigengesetzlichkeit überhaupt anerkannt wird“ (1983: 33). Ausdrücklich verweist Heller in diesem Zusammenhang auf Aristoteles, der ,den der heutigen Wissenschaft verwandtesten Typus einer politischen Wissenschaft [...] in Griechenland geschaffen“ habe, ,indem er den grundsätzlichen Schritt von der logischen und metaphysischen Spekulation zur Empirie tat" - ohne dass er das Ziel der „Erkenntnis des besten Staates aufgegeben hätte“ (24).

Jener grundsätzliche Schritt, von Voegelin klar erkannt, ist in dessen Augen gerade das Problematische, denn er führt letztlich zu einer Abwendung der Politikwissenschaft von den ordnungskonstitutiven Bewusstseinserfahrungen, und in der Neuzeit schließlich zu einer rein immanentistischen Wissenschaft, die für Voegelin Ausdruck moderner seelischer Unordnung und daher unfähig ist, ein adäquates Ordnungswissen hervorzubringen: „Wir haben keine noetische Ordnungswissenschaft mehr" (Voegelin 2005c: 334). Ob dieses Befundes fordert Voegelin die Politikwissenschaft auf, sich - wieder - auf die noetische oder die mystische Suche nach der Ordnung im Bewusstsein zu begeben, mithin platonische Ordnungswissenschaft zu werden (Voegelin 2005c: 351-354). Wenn aber für die Politikwissenschaft die Anerkennung der Politik als eigenständiger Seinsbereich konstitutiv bleibt, sie mithin wie von jeher aristotelisch sein muss, wird sie dieser Forderung distanziert gegenüberstehen müssen. Man darf annehmen, dass es diese sachlich begründete Distanz ist, die die Randständigkeit Voegelins zumindest in der Politikwissenschaft letztlich bestimmt.

Leider hat diese Marginalität zur Folge, dass die Möglichkeit, sich von Voegelin anregen und in konstruktiver Weise irritieren zu lassen, weithin nicht ergriffen wird. Denn der Platonismus ist zwar das Charakteristische (und Problematische) des Voegelin'schen Werkes, er steht aber keineswegs einer oft klaren Sicht auf politiktheoretische Grundfragen, auf machtpragmatische Zusammenhänge oder auf methodologische Probleme der Politikwissenschaft im Wege. Ganz im Gegenteil wird der sorgfältige Leser gerade in Ordnung und Geschichte immer wieder mit klugen Beobachtungen konfrontiert. ${ }^{17}$ So etwa wird jeder, der sich für die spezifische Rationalität politischen Handelns interessiert, gerne Voegelins Ausführungen in den Abschnitten über die Theorie, die Methode und die Form im Thukydides-Kapitel des

17 Solche Beobachtungen Voegelins führen gelegentlich direkt zu Methodenfragen. So konstatieren zum ersten Band von Ordnung und Geschichte auch Stefan Fietz und Hubertus Buchstein (2003: 104), er gebe „einen kräftigen Anstoß [...], die Methodendebatte in der politischen Ideengeschichtsschreibung nicht einfach ratlos einschlafen zu lassen“. Diese Feststellung gilt weit über die Ideengeschichtsschreibung hinaus. 
Michael Henkel

fünften Bandes der deutschen Ausgabe lesen (Voegelin 2003a: 228-256): Voegelins Darstellung, die zunächst daran erinnert, dass Thukydides ein politikwissenschaftliches Anliegen verfolgte (236), stellt nicht nur die im Peleponnesischen Krieg entfaltete Problematik der Perspektivität politischen Handelns vor, sondern sie hebt auch die von Thukydides diagnostizierte Spannung zwischen rationaler Machtausübung und rechtlichen Forderungen sowie den tragischen Charakter von Politik hervor, der sich in Notwendigkeiten zum Ausdruck bringt, denen die Handelnden nicht zu entfliehen vermögen. Gerade in der heutigen Lehre von den internationalen Beziehungen dürften Voegelins diesbezügliche Darlegungen angesichts etwa der jüngsten Debatten um das Konzept des Imperiums geeignet sein, frische Blicke auf die Gegenwart zu ermöglichen.

\section{Hitler und Deutschlands politische Kultur}

Voegelin selbst hat sich bei der politischen Diagnose seiner Gegenwart zumindest nach 1938 einige Zurückhaltung auferlegt. Allerdings war er keineswegs völlig enthaltsam, wofür das wichtigste Zeugnis in seinem Euvre die nunmehr erstmals hierzulande veröffentlichte Vorlesung über Hitler und die Deutschen (Voegelin 2006a) ist. Die Vorlesung hatte Voegelin im Sommersemester 1964 an der Münchener Universität gehalten. ${ }^{18}$ Obgleich bereits ein Verlagsvertrag für die Veröffentlichung abgeschlossen war, kam es nicht zur Publikation. Als einen Grund dafür hebt der Herausgeber des im Wesentlichen aus der Abschrift einer Tonbandaufnahme bestehenden Textes hervor, dass Voegelin damals die Weiterarbeit an den Studien zu Order and History wichtiger gewesen sei und dass er namentlich nicht ,das Leben eines öffentlichen Intellektuellen" (Henningsen 2006: 34, 11) habe führen wollen, er sich also aus der Beteiligung an aktuellen politischen Debatten herauszuhalten gesucht habe.

Dass er mit einer Veröffentlichung des Buches tatsächlich in den Fokus einer größeren (Fach-) Öffentlichkeit auch dann geraten wäre, wenn er den Text für die Publikation gründlich überarbeitet hätte, darf kaum bezweifelt werden: Es handelt sich nämlich in weiten Teilen um eine zutiefst polemische Generalabrechnung mit der politischen Kultur der Deutschen, einer Kultur, deren verrotteter Zustand die Herrschaft eines Hitler möglich gemacht und sich auch nach Überwindung des Hitlerregimes in ihrer irrationalen Substanz nicht verändert habe (Voegelin 2006a: 80).

Gerade auf diesen letzten Punkt kommt es Voegelin an: Es geht ihm um eine Diagnose des „Geisteszustand[es] einer Gesellschaft, in der Nationalsozialisten zur Macht kommen können“ (75). Da sich dieser Geisteszustand nach 1945 „nicht allzuviel geändert hat“ (76), finden sich in dem Text nicht nur historische Analysen über die geistige Welt des 19. und frühen 20. Jahrhunderts, sondern auch über das Grundgesetz, die juristische Rechtsstaatsdoktrin (221-238) und allgemein über das Phänomen der Realitätsverweigerung in der deutschen Gesellschaft des Jahres 1964. Der Nenner, auf den Voegelin seine Analysen bringt, ist

$18 \mathrm{Zu}$ der Veranstaltung gehörte auch die Vorlesung über Die Größe Max Webers, die Voegelin zu einer Veranstaltungsreihe der Universität anlässlich des 100. Geburtstages Webers beitrug; nachdem sie bereits an anderer Stelle veröffentlicht wurde, ist sie auch hier abgedruckt (Voegelin 2006a: 268-284). Außerdem ist der Text eines thematisch eng mit der Hitler-Vorlesung zusammenhängenden Vortrages Voegelins über Die deutsche Universität und die Ordnung der deutschen Gesellschaft von 1966 abgedruckt (285-316). 
die Dummheit (52-60, 84-90, 97-102, 105-109, passim). Die deutsche politische Kultur ist demnach gekennzeichnet durch Dummheit und damit einhergehend durch Illiteratentum und Analphabetismus (89) - Begriffe, die Voegelin ausdrücklich als diagnostische Instrumente (68) und als Erklärungsmodelle einführt.

Es ist nicht zu übersehen, dass die Analyseinstrumente und Erklärungen Voegelins nicht solche der politikwissenschaftlich-zeitgeschichtlichen Diagnose sind; und in der Tat distanziert sich Voegelin deutlich von der Zeitgeschichtsforschung. Deren Methoden hält er für die wissenschaftliche Auseinandersetzung mit Geschichte und Gegenwart für defizitär, und zwar unter anderem deshalb, weil sie die Vergangenheit nicht von vornherein als Gegenwartsproblem, gewissermaßen als eigene Frage behandle (74 f.; dazu Henningsen 2006: 21-29). Zweifellos vermag Voegelin mit seinen Analyseinstrumenten den intellektuellen Primitivismus mancher Auswüchse bürgerlicher Weltanschauung des 19. Jahrhunderts beispielsweise im Werk von Ernst Haeckel treffend zu entlarven, und unstrittig dürfte der in diesem Zusammenhang erfolgende - allerdings nicht originelle - Nachweis des Primitivismus' von Hitlers Weltanschauung sein (Voegelin 2006a: 114-150). Vor diesem Hintergrund ist auch Voegelins Kritik an dem Historiker Percy Ernst Schramm kaum verfehlt: Schramm hatte 1963 Hitlers Tischgespräche veröffentlicht und für dieses Buch eine Charakterisierung Hitlers vorgenommen, die tatsächlich überaus naiv und mit wenig politischem Sinn verfasst war. Diese Darlegungen Schramms hatten seinerzeit eine öffentliche Debatte zur Folge, und sie bildeten letztlich für Voegelin den Anlass, die Hitler-Vorlesung zu halten (Henningsen 2006: 12 f.; Voegelin 2006a: 49-51): Schramm galt Voegelin als repräsentativer Analphabet, an dem sich das Desaster des deutschen Geisteszustandes paradigmatisch veranschaulichen ließ.

Die Stärke der Voegelin'schen Einlassungen mag man in der Kritik eines unkritischen bürgerlichen Bewusstseins sehen. Aber diese Kritik ist ihrerseits in mehrfacher Hinsicht problematisch. Zunächst folgt aus ihr nichts politisch Greifbares. Am Ende der Vorlesung geht es Voegelin in großer Allgemeinheit um die Wiedergewinnung von Realität und Ordnung. Das heißt im Wesentlichen, dass er die Anerkennung „der“ Realität einfordert, also letztlich die Anerkennung der ordnenden Wirklichkeit der Transzendenz, der „Existenz des Menschen in seiner Präsenz unter Gott“ (Voegelin 2006a: 69). Eine entsprechende „Umkehr" gilt Voegelin als das entscheidende Mittel zur Überwindung des diagnostizierten Realitätsverlustes. Dass hieraus aber keinerlei politische Konsequenzen abzuleiten sind, liegt auf der Hand: Weder impliziert die Anerkennung der Transzendenz irgendein politisches „Programm" - etwa die Befürwortung einer republikanischen Ordnung -, noch ist zu erkennen, dass und wie sie vor politischem Irrtum zu schützen vermöchte.

An diesem Punkt wird eine bestimmte Seite des Voegelin'schen Platonismus offenbar: Das für diesen charakteristische Fehlen der Anerkennung eines eigenständigen Politikbegriffs verführt zu einem unpolitischen Moralismus in der Betrachtung von Politik, Gesellschaft und Geschichte. Jedenfalls in der Hitler-Vorlesung ist Voegelin der Verführung eines solchen Moralismus erlegen, was sein politisches Urteilsvermögen in vielen Punkten erheblich eintrübt (siehe etwa 238) Allerdings stehen auch in der Hitler-Vorlesung neben allerhand fragwürdigen Behauptungen manch interessante und anregende Feststellungen, die einen Kontrast zu jenem mangelnden politischen Urteil darstellen. Dies gilt etwa für Voegelins Bemerkungen über die demokratische Tugend des Kompromisses ( $83 \mathrm{f}$.) oder für eine kluge 
Michael Henkel

Beobachtung über Hitlers Herrschaft: Voegelin erläutert, dass für den Realitätsverlust eine Haltung charakteristisch sei, die er in einem Satz von Novalis ausgedrückt sieht: „Die Welt soll sein, wie ich es will““. Diesem Novalis-Zitat lässt Voegelin die Feststellung folgen: „In diesem Satz haben Sie in nuce das ganze Problem Hitlers“ (86). Hierin steckt sicher einige Wahrheit.

Vor dem Hintergrund des inhaltlich ambivalenten und z. T. fragwürdigen Charakters sowie der überschießenden Polemik der Vorlesung darf man vermuten, dass Voegelin diese vielleicht auch deshalb nicht veröffentlichen wollte, weil er ihre sachliche Angreifbarkeit erkannte. Als Forschungsdokument zur Ergründung des Nationalsozialismus wie der deutschen politischen Kultur jedenfalls wird man die Vorlesung nur in sehr beschränktem Umfang ansehen dürfen.

\section{Die Krise der Moderne}

Voegelins Urteil über Hitler und die deutsche Gesellschaft liegt ganz in der Konsequenz seines Verständnisses der Neuzeit als im Kern irrationalem Zeitalter. Die in der ursprünglichen Anlage von Order and History geplante zusammenhängende Darstellung des neuzeitlichen Ordnungs- und Geschichtsdenkens hat Voegelin wie bemerkt nicht vorgelegt: In seinen substanziellen Themen geht Order and History nicht über die Zeit der Spätantike hinaus. Allerdings findet sich in den Bänden eine Vielzahl eingeschobener, oft eher beiläufiger Hinweise und Erläuterungen mit Blick auf das neuzeitliche Denken, ${ }^{19}$ und die genaue Lektüre dieser Stellen bestätigt, dass es Voegelin in seinem magnum opus stets auch um ein Verständnis der Neuzeit und der Gegenwart geht. Dies gilt insbesonderere für die Bände von 1974 und 1987 (Winterholler 2006: 8 f.).

Signifikant ist diesbezüglich, dass sich Voegelin im abschließenden Band in einem langen Kapitel mit Hegel als repräsentativem modernen Denker ausführlich auseinandersetzt (Voegelin 2004c: 67-85). Bereits im Ökumenischen Zeitalter war Hegel als der am häufigsten genannte moderne Denker überaus präsent - eine Folge des Umstandes, dass Voegelin in dem Philosophen immer klarer einen herausragenden geschichtsphilosophischen Gegenspieler erkannte. Daher widmete er dem bedeutenden Schwaben seit den späten fünfziger Jahren eine verstärkte Aufmerksamkeit. Seine gründlichste Auseinandersetzung mit Hegel legte er 1971 in einem umfangreichen Aufsatz vor, dessen zuerst 1999 in den Occasional Papers erschienene Übersetzung (Hegel - Eine Studie über Zauberei) nun zusammen mit einer weiteren bewusstseinsphilosophischen Studie aus den siebziger Jahren (Weisheit und die Magie des Extrems: Eine Meditation) vom Passagen-Verlag veröffentlicht wurde (Voegelin 2006b).

Die beiden für den späten Voegelin charakteristischen Texte zeigen in ihrer Kombination deutlich, wie sich Voegelins Verständnis der von ihm konstatierten modernen Realitätsverweigerung aus seiner konstruktiven mystischen Position ergibt. In seiner Kritik modernen Denkens sucht er dabei immer sensibler die Erfahrungsgrundlagen ausfindig zu machen, die einerseits ein Bewusstsein der Realität auch bei modernen Denkern erkennen lassen, die aber andererseits in den dogmatischen Symbolisierungen dieser Denker verzerrt und ideologisch deformiert werden, und zwar in dem Bestreben, die in der Transzendenz begründete Realität

19 Etwa Voegelin 2002a: 74; 2002b: 31-42; 2002c: 92; 2003a: 121; 2004b: 82; 2005a: 41-43. 
zu beherrschen und die Geschichte wie die Philosophie gewissermaßen zum Stillstand zu bringen - ein Bestreben, das Voegelin als spekulative Zauberei qualifiziert.

Das hierin beschlossene Verdikt darf nicht darüber hinwegtäuschen, dass Voegelin Hegel mit einiger Hochachtung begegnet und er sich daher auf dessen Werk tatsächlich einzulassen sucht (Winterholler 2006: 15-25). Darin unterscheidet sich seine Hegel-Deutung etwa von dem grotesken Hegelbild, das im 20. Jahrhundert wirkmächtig namentlich von Karl Popper gezeichnet wurde. Gleichwohl kann man nicht verkennen, dass Voegelins Lesart und die darin beschlossene Kritik ganz und gar von seinen eigenen, zu Idiosynkratien nötigenden bewusstseinsphilosophischen Kategorien und seinem eigenwilligen Philosophieverständnis (Voegelin 2004c: 68) abhängt. Das führt schließlich dazu, dass auch er Hegels Intention ebenso wie dessen Begriffe letztlich verfehlt, was schon äußerlich daran erkennbar ist, dass das zentrale Konzept der praktischen Philosophie Hegels - das der Freiheit - in Voegelins Interpretation keine Rolle spielt (dazu Henkel 1998: 174-177).

Der Hegelstudie vergleichbare Auseinandersetzungen mit neuzeitlichen oder zeitgenössischen Denkern finden sich in Voegelins späten Texten allenfalls in Anamnesis, ansonsten in der konzise zusammenhängenden und programmatischen Darstellung modernen Denkens, die er im Drama des Menschseins präsentiert (2007c: 71-96). Im Übrigen bleibt es bei den verstreuten Ausführungen in Order and History und in verschiedenen Aufsätzen. Für ausführlichere und argumentativ zusammenhängende Darlegungen zum neuzeitlichen Denken aus Voegelins Feder ist man mithin auf seine älteren Arbeiten verwiesen, namentlich auf die Neue Wissenschaft, die Antrittsvorlesung über Wissenschaft, Politik und Gnosis (Voegelin 1959b; 1999: 57-90) sowie auf einige Aufsätze aus den 40er- und 50er-Jahren. Bei letzteren handelte es sich teilweise um Auszüge aus einer im Ganzen von Voegelin selbst nicht publizierten History of Political Ideas, einem Monumentalwerk, an dem er in den 40er- bis in die Mitte der 50er-Jahre gearbeitet und das er bis zu einem weithin druckreifen Manuskript fertiggestellt hatte, bevor er die Konzeption des Werkes verwarf und einen Neuanlauf unternahm, den er zuerst in der Neuen Wissenschaft präsentierte (Henkel 1998: 25 ff.; Opitz 2002: 239-263). Im Unterschied zu Ordnung und Geschichte gilt der überwiegende Teil der History einer Darstellung neuzeitlichen Denkens, die inzwischen vollständig nachgelesen werden kann: Die History ist im Rahmen der Collected Works in acht Bänden (Bde. 19 bis 26; Columbia, London 1997-1999) vollständig veröffentlicht, wobei fünf Bände (Bde. 22-26) der Neuzeit gewidmet sind.

Das Manuskript der History blieb für Voegelin ein Materialfundus, auf den er auch später zurückgriff. Darüber hinaus scheinen Teile des Werkes für ihn aber ihre Rechenschaftsfähigkeit nicht verloren zu haben. So findet sich in Anamnesis ein Kapitel, das der History entnommen ist, nämlich der zuerst 1946 in den USA publizierte Text über Bakunins Beichte (Voegelin 2005c: 223-238). Die Studie, die Bakunin als Repräsentanten eines modernen Revolutionarismus und als Wegbereiter der „Politik Lenins und Stalins“ (236) zeigt, war im Manuskript der History ein Teil des letzten Bandes, in dem Voegelin sich mit der Krise der jüngeren Moderne auseinandersetzt. Diese Studie scheint er noch Mitte der 50er-Jahre als den Schlussband von Order and History in dessen ursprünglicher Konzeption (als Bd. VI. The Crisis of Western Civilization) vorgesehen zu haben (Opitz 2008: 17). Und selbst nachdem Voegelin diese Konzeption preisgegeben hatte, hielt er den Band der Veröffentlichung 
für wert. So konnte ihn John H. Hallowell 1975 unter dem Titel From Enlightenment to Revolution (mit kleinen Modifikationen gegenüber dem originalen Manuskript) herausgeben. Voegelin mag der Publikation des inzwischen über 25 Jahre alten Textes auch deshalb zugestimmt haben, weil er zu dieser Zeit vermutlich sah, dass es zu einer umfassenderen Behandlung neuzeitlichen Denkens im Rahmen von Order and History nicht mehr kommen würde. Der Text ist jetzt in der Periagoge-Reihe unter dem Titel Die Krise. Zur Pathologie des modernen Geistes erschienen (Voegelin 2008). Neben einzelnen für Voegelins intellektuelle Entwicklung besonders wichtigen Teilen und Kapiteln aus dem History-Manuskript, die in den vergangenen Jahren von Opitz herausgegeben wurden, ${ }^{20}$ liegt damit nun ein Band der History vollständig auch in deutscher Sprache vor.

Der voluminöse Band, der von nicht weniger als sechs Übersetzerinnen und Übersetzern übertragen und mit hilfreichen textkritischen Hinweisen versehen wurde, setzt sich mit Helvetius, den verschiedenen Strömungen des klassischen Positivismus, und hier insbesondere mit Comte („die erste große Gestalt der Krise des Westens“, Voegelin 2008: 195), ferner mit Bakunin sowie mit Marx auseinander. Dabei wird in den diesen Themen bzw. Autoren gewidmeten umfangreichen Kapiteln eine große Zahl von Denkern diskutiert, um Kontexte und Zusammenhänge deutlich werden zu lassen. So thematisiert Voegelin etwa d'Alambert, die Marquise du Chatelet, Bossuet, Condorcet, Feuerbach, Hegel, Lenin, Locke oder Voltaire in mehr oder minder großer Ausführlichkeit. Diese Liste zeigt, dass Voegelin nicht allein solche Autoren diskutiert, die üblicherweise in ideengeschichtlichen Darstellungen behandelt, sondern auch solche, die sonst eher vernachlässigt werden, obgleich sie für die neuzeitliche Denkentwicklung keineswegs unbedeutend sind. So erhält man bei ihm manch interessante Einblicke in „Zwischenwelten“ (Ernst Bloch) der Philosophiegeschichte.

In der Ausmalung seines Panoramas des modernen Geistes schüttet Voegelin das Füllhorn seines Wissens vor dem Leser aus und präsentiert eine große Zahl ebenso interessanter wie bisweilen überaus eigenwilliger Interpretationen sowie verstreute Überlegungen zur Eigenart und Methodik einer Ideengeschichte (etwa 106 f.). So ist es beispielsweise überaus lohnend, der Auseinandersetzung mit dem Marx'schen Dialektikbegriff zu folgen (367-387): Der an Platons Dialektik geschulte Voegelin kann im Ausgang von der Feststellung, der Begriff des dialektischen Materialismus sei ,eine contradictio in adjecto“ (368), durchaus überzeugend darlegen, dass Marx ein intellektueller Schwindler war (372). Die Argumentation zeigt, dass dieses Urteil weniger Polemik enthält, als es zunächst scheinen mag - was man selbst dann konstatieren kann, wenn man Voegelins weitergehender Ansicht über eine spezifisch moderne Pathologie des Geistes bei Marx nicht beistimmt. Wie in diesem Beispiel regt Die Krise auch sonst oft zum Weiterdenken und zum Perspektivenwechsel an, auch, wo man Voegelin schließlich nicht folgen wird.

Der Krisis-Band - und damit die History im Ganzen - sollte ursprünglich mit einem Kapitel über Nietzsche enden, das Voegelin nicht mehr verfasst hat (Opitz 2008: 18 f.; 2007c: 162 f.). Indes hat er 1944 zwei Aufsätze über Nietzsche geschrieben, von denen nur einer (Nietzsche, die Krise und der Krieg) veröffentlicht wurde, während der andere (Pascal

20 Es handelt sich dabei vor allem um solche Abschnitte der History, in denen sich Voegelin mit modernen Denkern auseinandersetzt, welchen er sich intellektuell verbunden fühlt (Voegelin 2003b; 2003c; 2004d) oder um eine systematisch angelegte Interpretation historischer Kontinuitätslinien (Voegelin 1994). 
und Nietzsche) bis nach seinem Tod ungedruckt blieb. Diese Texte liegen nun ebenfalls vollständig auf Deutsch vor: Im Berliner Verlag Matthes \& Seitz sind sie gemeinsam unter dem Titel Das Jüngste Gericht: Friedrich Nietzsche und mit einem ausführlichen Nachwort des Herausgebers Opitz in einem ästhetisch besonders ansprechenden Büchlein veröffentlicht worden (Voegelin 2007d).

Die beiden Aufsätze können ohne Weiteres als selbstständige Nietzsche-Interpretationen unabhängig vom Kontext des Voegelin'schen Werkes gelesen werden. Sie zeigen Nietzsche nicht nur als herausragenden Diagnostiker, sondern zugleich als bedeutendes Symptom der Krise der Moderne. Das hier gezeichnete Nietzsche-Bild lässt eine - wenn auch distanzierte Hochachtung für Nietzsche klar erkennen, die nach dem Krieg allerdings einer dezidierten Kritik gewichen ist. Beides, Hochachtung und spätere Zurückweisung beruhen indes auf demselben Fundament: Das Umwertungs-Projekt Nietzsches, durchaus auf platonischem Boden gewachsen, ist für Voegelin ein solches, das „nicht bloß ein neues Moralsystem“, sondern vielmehr eine ,radikale Persönlichkeitsreform“ (25) verlange, und eine solche Reform ist es letztlich, die Voegelin selbst unter dem Titel der Umkehr (periagoge) einfordert.

Die spezifische Differenz zwischen beiden Denkern besteht nicht etwa darin, dass Voegelin zur Reform den Weg der Mystik beschreitet. Denn auch Nietzsche gilt ihm ausdrücklich als Mystiker (69, 80, 117, 127, 129). Der nach 1944 für Voegelin an Gewicht gewinnende Unterschied besteht vielmehr darin, dass die Mystik Nietzsches eine radikal innerweltliche ist, die sich der Transzendenz aktiv verschließt. So wird die immanentistische Mystik zur Quelle des Gottesmordes (Voegelin 1999: 92, 96-99) und bringt den Aktivismus des Willens zur Macht hervor - einen, wie es an anderer Stelle heißt, ,magischen Aktivismus“ (Voegelin 2006b: 31) der Selbsterlösung (Voegelin 2007b: 140). Es ist der Aktivismus einer Verdrängung der Realität zugunsten einer zweiten Realität, einer Traumwelt, die jene Ungeheuer gebiert, welche sich schließlich in den Gewaltexzessen der totalitären Bewegungen Bahn gebrochen haben. In dieser Perspektive erweist sich, dass man in Nietzsche den eigentlichen Gegenspieler Voegelins sehen darf (siehe auch Ottmann 1996: 198).

Das Stichwort der Gewalt verweist auf ein von der Forschung bisher noch vernachlässigtes Thema, das sich seit 1938 untergründig wie ein roter Faden durch Voegelins Arbeiten zieht: Immer wieder sucht Voegelin in seinem Werk jene geistigen Kräfte zu identifizieren, die in der Geschichte zur Legitimation von Gewalt führten oder deren innere Logik die Anwendung von Gewalt fordert. So treten etwa die Kriege des Alten Testaments (Voegelin 2005b: 38-40, 123), die Gewaltextasen mittelalterlicher und besonders neuzeitlicher Sektenbewegungen (Voegelin 1994: 73-75), die Gewaltrechtfertigungen im Koran (Voegelin 2004a: 184-187) oder die Gewaltlegitimationen moderner politischer Bewegungen des Westens (Voegelin 1999: 100; 2008: 418) ins Blickfeld. Worauf es Voegelin dabei offenkundig ankommt, ist es, solche Gewalt zu diskreditieren, die sich als Ausdruck geistiger Unordnung erweisen lässt. Wie tragfähig die entsprechenden Analysen im Einzelnen sind, bedarf gewiss genauerer Untersuchung. Dabei könnte sich durchaus herausstellen, dass Voegelins bewusstseinsphilosophische Überlegungen - wie das Konzept der „existenziellen Revolte“ (Voegelin 2004c: 44-47) - einen womöglich gewichtigeren Beitrag etwa zur Erklärung islamistischen Terrors zu liefern vermögen als beispielsweise landläufige Hinweise auf wirtschaftliche Unterentwicklung als dessen Ursache. 
Hier zeigt sich das Werk Voegelins abermals als anregend und als überaus aktuell. Wie eine politikwissenschaftliche Voegelin-Rezeption mit den im Vorstehenden mehrfach angesprochenen Anregungen hinsichtlich aktueller Fragestellungen umgehen und was sie von Voegelin lernen kann, bleibt im einzelnen Fall zu prüfen. Eine prinzipielle Orientierung mögen dabei indes die Worte geben, mit denen Jan Assmann seinen Kommentar zum ersten Band von Ordnung und Geschichte abschließt: „Die [...] Kritikpunkte sollen vor allem zeigen, dass sich mit den von Voegelin eingeführten Begriffen und auf den von ihm angelegten Argumentationslinien hervorragend arbeiten und weiterkommen lässt. Voegelin ist [...] kein Autor, der sich zu einer alexandrinischen Kanonisierungs- und Auslegungskultur im Stile der Max-Weber-Forschung eignet, sondern ein Bahnbrecher und Anreger, der zu Weiterforschen und Weiterdenken, Vertiefen, Widerlegen, Zustimmung und Widerspruch heraufordert“ (2002: 224).

\section{Literatur}

Assmann, Jan, 2002: Nachwort, in: Voegelin 2002a, 213-224.

Backes, Uwe/Jesse, Eckhard, 1992: Totalitarismus und Totalitarismusforschung. Zur Renaissance einer lange tabuisierten Konzeption, http:/www.extremismus.com/texte/total1.htm (Stand: 19.01.08). [Printversion in: dies. (Hrsg.), Extremismus \& Demokratie, Bd. 4, Bonn (1992)].

Bergbauer, Harald, 2002: Bausteine der Voegelin-Forschung: Occasional Papers, in: ZfP 49, 96-106.

Blößner, Norbert, 2001: „The Encomium of a Noble Man“. Anmerkungen zu Eric Voegelins Politeia-Interpretation, München (Occasional Papers XXI).

Caringella, Paul/Weiss, Gilbert, 2004a: Vorbemerkung der Herausgeber, in: Voegelin 2004c, 15-16.

Caringella, Paul/Weiss, Gilbert, 2004b: Nachwort, in: Voegelin 2004c, 137-175.

Duso, Giuseppe, 2006: Die moderne politische Repräsentation: Entstehung und Krise des Begriffs, Berlin.

Fietz, Stefan/Buchstein, Hubertus, 2003: [Besprechung] Eric Voegelin, Ordnung und Geschichte, Band 1: Die kosmologischen Reiche des Alten Orients - Mesopotamien und Ägypten, in: PVS 44, 102-104.

Flashar, Hellmut, 2001: Dekomposition einer mythischen Philosophie. Eric Voegelin über Aristoteles, München (Occasional Papers XXII).

Flashar, Hellmut, 2003: [Besprechung] Eric Voegelin, Aristoteles, in: Gymnasium 110, 69-71.

Flashar, Hellmut, 2005: [Besprechung] Ordnung und Geschichte, Bd. 4/Bd. 5, in: ZfP 52, 123-125.

Gebhardt, Jürgen, 1982: Toward the Process of Universal Mankind: The Formation of Voegelin's Philosophy of History, in: Ellis Sandoz (Hrsg.), Eric Voegelin's Thought. A Critical Appraisal, Durham, 67-86. 
Gebhardt, Jürgen, 2003: Voegelin und das Politische, in: Michael Ley/Heinrich Neisser/ Gilbert Weiss (Hrsg.), Politische Religion? Politik, Religion und Anthropologie im Werk von Eric Voegelin, München, 199-208.

Haas, Alois M., 2002: Die Problematik von Sprache und Erfahrung in der deutschen Mystik, in: Werner Beierwaltes/Hans Urs von Balthasar/Alois M. Haas, Grundfragen der Mystik, Einsiedeln (2. Aufl.), 75-106.

Hartenstein, Friedhelm/Jeremias, Jörg, 2005: Zur Einführung, in: Voegelin 2005a, 11-16.

Heller, Hermann, 1983: Staatslehre, Tübingen (6. Aufl., zuerst 1934).

Henkel, Michael, 1998: Eric Voegelin zur Einführung, Hamburg.

Henningsen, Manfred, 2006: „Eine Mischung aus Schlachthof und Klapsmühle“. Einleitung zu Eric Voegelin, Hitler und die Deutschen, in: Voegelin 2006a, 9-38.

Hollweck, Thomas A., 1999: Das gnostische Wesen der Moderne: Die Zukunft einer These, in: Voegelin 1999, 144-159.

Horn, Christoph, 2001: Kontinuität, Revision oder Weiterentwicklung? Das Verhältnis von Politeia, Politikos und Nomoi bei Eric Voegelin und in der aktuellen Forschung, München (Occasional Papers XXIII).

Hughes, Glenn/McNight, Stephen A./Price, Geoffrey L. (Hrsg.), 2001: Politics, Order and History. Essays on the Work of Eric Voegelin, Sheffield.

Kuhn, Helmut, 1960: Heilsgeschichte und Weltgeschichte, in: Philosophische Rundschau 8, 122-126.

Lübbe, Hermann, 2003: Zustimmungsfähige Modernität. Gründe einer marginal verbliebenen Rezeption Eric Voegelins, München (Occasional Papers XXXIV).

Machinist, Peter, 2002: Mesopotamien in Eric Voegelins Ordnung und Geschichte, in: Voegelin 2002a, 177-212.

Maier, Hans, 1995: Politische Religionen. Die totalitären Regime und das Christentum, Freiburg u. a.

Maier, Hans (Hrsg.), 1996: Totalitarismus und Politische Religionen. Konzepte des Diktaturvergleichs, Bd. I, Paderborn u. a.

Maier, Hans (Hrsg.), 2003: Totalitarismus und Politische Religionen. Konzepte des Diktaturvergleichs, Bd. III, Deutungsgeschichte und Theorie, Paderborn u. a.

Maier, Hans/Schäfer, Michael (Hrsg.), 1997: Totalitarismus und Politische Religionen. Konzepte des Diktaturvergleichs, Bd. II, Paderborn u. a.

Meier, Christian, 1995: Die Entstehung des Politischen bei den Griechen, Frankfurt a. M. (3. Aufl., zuerst 1980).

Mommsen, Hans, 1962: Zum Verhältnis von Politischer Wissenschaft und Geschichtswissenschaft in Deutschland, in: Vierteljahreshefte für Zeitgeschichte 10, 341-372.

Opitz, Peter J., 1989: Spurensuche - Zum Einfluß Eric Voegelins auf die politische Wissenschaft in der Bundesrepublik Deutschland, in: ZfP 36, 235-250.

Opitz, Peter J., 1999: Die Gnosis-These - Anmerkungen zu Eric Voegelins Interpretation der westlichen Moderne, in: Voegelin 1999, 7-35.

Opitz, Peter J., 2000: Der Weg des Himmels. Zum Geist und zur Gestalt des politischen Denkens im klassischen China, München. 
Opitz, Peter J., 2002: Vom „System der Staatslehre“ zur „Philosophie der Politik und der Geschichte": Zur Entstehungsgeschichte von Eric Voegelins Order and History, in: Voegelin 2002a, 225-286.

Opitz, Peter J. (Hrsg.), 2005: Voegeliniana. Veröffentlichungen von und zu Eric Voegelin, München (Occasional Papers XLVI, 2. Aufl.).

Opitz, Peter J., 2007a: Eric Voegelins Politische Religionen. Kontexte und Kontinuitäten, in: Voegelin 2007a, 69-150.

Opitz, Peter J., 2007b: The New Science of Politics - Versuch einer geistigen und werksgeschichtlichen Ortsbestimmung, in: Voegelin 2007b, 203-263.

Opitz, Peter J., 2007c: Eric Voegelin, die „Krise“ und Friedrich Nietzsche, in: Voegelin 2007d, 139-167.

Opitz, Peter J., 2008: Vorwort des Herausgebers, in: Voegelin 2008, 15-22.

Ottmann, Henning, 1996: Das Spiel der Masken. Nietzsche im Werk Eric Voegelins, in: Nietzsche-Studien 25, 191-199.

Pitkin, Hanna F., 1972: The Concept of Representation, Berkeley u. a. (zuerst 1967).

Price, Geoffrey L., 2000: Eric Voegelin: International Bibliography 1921-2000, München.

Schütrumpf, Eckart E., 2001: Eric Voegelins Deutung der aristotelischen Politik in Order and History, München (Occasional Papers XXIV).

Seitschek, Hans Otto, 2003: Exkurs: Eric Voegelins Konzept der „Gnosis“, in: Hans Maier (Hrsg.), Totalitarismus und Politische Religionen. Konzepte des Diktaturvergleichs, Bd. III, Deutungsgeschichte und Theorie, Paderborn u. a., 237-245.

Siegel, Achim (Hrsg.), 1998: Totalitarismustheorien nach dem Ende des Kommunismus, Köln/Weimar.

Söllner, Alfons/Walkenhaus, Ralf/Wieland, Karin (Hrsg.), 1997: Totalitarismus. Eine Ideengeschichte des 20. Jahrhunderts, Berlin.

Voegelin, Eric, 1959a: Die Neue Wissenschaft der Politik. Eine Einführung, München.

Voegelin, Eric, 1959b: Wissenschaft, Politik und Gnosis, München.

Voegelin, Eric, 1977: Die Neue Wissenschaft der Politik. Eine Einführung, Salzburg (3. Aufl.).

Voegelin, Eric, 1985: Religionsersatz. Die gnostischen Massenbewegungen unserer Zeit, Tutzing (zuerst 1960).

Voegelin, Eric, 1988a: Äquivalenz von Erfahrungen und Symbolen in der Geschichte, in: Voegelin 1988b, 99-126 (zuerst 1970).

Voegelin, Eric, 1988b: Ordnung, Bewusstsein, Geschichte. Späte Schriften - eine Auswahl, hrsg. von Peter J. Opitz, Stuttgart.

Voegelin, Eric, 1991: Die Neue Wissenschaft der Politik. Eine Einführung, hrsg. von Peter J. Opitz; mit einem Nachwort des Herausgebers und einer Bibliographie, Freiburg/ München (4. Aufl.).

Voegelin, Eric, 1993: Die politischen Religionen, hrsg. und mit einem Nachwort von Peter J. Opitz, München.

Voegelin, Eric, 1994: Das Volk Gottes. Sektenbewegungen und der Geist der Moderne, hrsg., eingeleitet und mit einem Essay von Peter J. Opitz, München.

Voegelin, Eric, 1997: Der autoritäre Staat. Ein Versuch über das autoritäre Staatsproblem, Wien/New York (zuerst 1936). 
Voegelin, Eric, 1999: Der Gottesmord. Zur Genese und Gestalt der modernen politischen Gnosis, hrsg. und eingeleitet von Peter J. Opitz, mit einem Nachwort von Thomas Hollweck, München.

Voegelin, Eric, 2001: Ordnung und Geschichte, Bd. 7, Aristoteles, hrsg. von Peter J. Opitz, Paderborn/München.

Voegelin, Eric, 2002a: Ordnung und Geschichte, Bd. 1, Die kosmologischen Reiche des Alten Orients: Mesopotamien und Ägypten, hrsg. von Jan Assmann, Paderborn/München.

Voegelin, Eric, 2002b: Ordnung und Geschichte, Bd. 4, Die Welt der Polis: Gesellschaft, Mythos und Geschichte, hrsg. von Jürgen Gebhardt, Paderborn/München.

Voegelin, Eric, 2002c: Ordnung und Geschichte, Bd. 6, Platon, hrsg. von Dietmar Herz, Paderborn/München.

Voegelin, Eric, 2003a: Ordnung und Geschichte, Bd. 5, Die Welt der Polis: Vom Mythos zur Philosophie, hrsg. von Jürgen Gebhardt, Paderborn/München.

Voegelin, Eric, 2003b: Giambattista Vico - La Scienza Nuova, hrsg. und mit einem Nachwort von Peter J. Opitz, mit einem Nachwort von Stephan Otto, München.

Voegelin, Eric, 2003c: Jean Bodin, hrsg. und mit einem Nachwort von Peter J. Opitz, München.

Voegelin, Eric, 2004a: Ordnung und Geschichte, Bd. 8, Das Ökumenische Zeitalter: Die Legitimität der Antike, hrsg. von Thomas Hollweck, Paderborn/München.

Voegelin, Eric, 2004b: Ordnung und Geschichte, Bd. 9, Das Ökumenische Zeitalter: Weltherrschaft und Philosophie, hrsg. von Manfred Henningsen, Paderborn/München.

Voegelin, Eric, 2004c: Ordnung und Geschichte, Bd. 10, Auf der Suche nach Ordnung, hrsg. von Paul Caringella/Gilbert Weiss, Paderborn/München.

Voegelin, Eric, 2004d: Schelling, hrsg. und mit einem Nachwort von Peter J. Opitz, München (Occasional Papers XLV).

Voegelin, Eric, 2005a: Ordnung und Geschichte, Bd. 2, Israel und die Offenbarung: Die Geburt der Geschichte, hrsg. von Jörg Jeremias und Friedhelm Hartenstein, Paderborn/ München.

Voegelin, Eric, 2005b: Ordnung und Geschichte, Bd. 3, Israel und die Offenbarung: Moses und die Propheten, hrsg. von Jörg Jeremias und Friedhelm Hartenstein, Paderborn/München.

Voegelin, Eric, 2005c: Anamnesis. Zur Theorie der Geschichte und Politik, Freiburg/ München (zuerst 1966).

Voegelin, Eric, 2006a: Hitler und die Deutschen, hrsg. von Manfred Henningsen, München.

Voegelin, Eric, 2006b: Mysterium, Mythos und Magie. Bewußtseinsphilosophische Meditationen, hrsg. und mit einem Vorwort von Peter J. Opitz, Wien.

Voegelin, Eric, 2007a: Die politischen Religionen, hrsg. und mit einem Nachwort versehen von Peter J. Opitz, Paderborn/München (3. Aufl.).

Voegelin, Eric, 2007b: Die Neue Wissenschaft der Politik. Eine Einführung, hrsg. von Peter J. Opitz, mit einem Nachwort des Herausgebers und einer Bibliographie, Paderborn/ München.

Voegelin, Eric, 2007c: Das Drama des Menschseins. Die Walter Turner Candler Lectures, hrsg. und mit einem Nachwort von Peter J. Opitz, Wien.

Voegelin, Eric, 2007d: Das Jüngste Gericht: Friedrich Nietzsche, hrsg. und kommentiert von Peter J. Opitz, Berlin. 
Voegelin, Eric, 2008: Die Krise. Zur Pathologie des modernen Geistes, hrsg. von Peter J. Opitz, Paderborn/München.

Weiss, Gilbert, 2003: Die europäische Rezeption Eric Voegelins, in: Michael Ley/Heinrich Neisser/Gilbert Weiss (Hrsg.), Politische Religion? Politik, Religion und Anthropologie im Werk von Eric Voegelin, München, 27-46.

Winterholler, Helmut, 2006: Egophanische Revolte und existenzieller Widerstand. Studien zu Eric Voegelins In Search of Order, München (Occasional Papers LII).

Korrespondenzanschrift:

Dr. Michael Henkel

Friedrich-Schiller-Universität Jena

Institut für Politikwissenschaft

07737 Jena

E-Mail: michael.henkel@uni-jena.de

Web: http://www.mhenkel.de

\title{
1.500 deutsche Bürgermeister - die Analyse
}

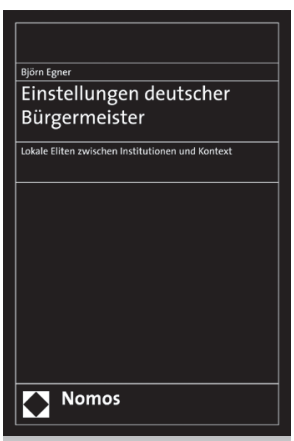

\author{
Einstellungen deutscher Bürgermeister \\ Lokale Eliten zwischen Institutionen und Kontext \\ Von Björn Egner \\ 2007, 261 S., brosch., 49,- €, ISBN 978-3-8329-3155-1
}

Die Studie untersucht erstmals, ob Einstellungen lokaler politischer Eliten durch institutionelle Strukturen und Besonderheiten von Gemeinden beeinflusst werden. Zugleich liefert die Untersuchung einen wertvollen Überblick über soziale Zusammensetzung und politische Orientierung von über 1.500 deutschen Bürgermeistern sowie ihre Sichtweisen auf Fragen lokaler Politik.

Bitte bestellen Sie bei Ihrer Buchhandlung oder bei Nomos | Telefon 07221/2104-37 | Fax -43 | www.nomos.de | sabine.horn@nomos.de

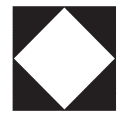

Nomos 